\title{
SFCW Microwave Radar for In-Door Fall Detection
}

\author{
M. Mercuri ${ }^{1}$, D. Schreurs ${ }^{1}$, and P. Leroux ${ }^{2,3}$ \\ ${ }^{1}$ K.U.Leuven, Div. ESAT-TELEMIC, Leuven, Belgium \\ ${ }^{2}$ K.U.Leuven, Div. ESAT-MICAS, Leuven, Belgium \\ ${ }^{3}$ K.H.Kempen, Div. IBW-RELIC, Geel, Belgium
}

\begin{abstract}
The use of a Stepped-Frequency Continuous Wave (SFCW) radar is proposed for non-invasive fall and vital signs detection. A fall in principle involves changes both in position and in speed. Measurements have been performed with the radar fixed both on the wall and on the ceiling. In both situations, position and speed of a target have been measured with good accuracy. By combining this information a fall can be properly detected, distinguishing the fall from both walking and sitting movements. The results show the feasibility of this approach. Moreover, the results demonstrate that vital signs can be monitored also.
\end{abstract}

Index Terms - Fall detection, health monitoring, radar remote sensing, SFCW radar.

\section{INTRODUCTION}

The elderly population (65 years and older) in the world has been steadily increasing. The situation has resulted in a growing need for healthcare approaches that emphasize routine long-term monitoring. Monitoring is of primary interest in the home situation [1]. As there is a general shortage of nursing homes, there is the incentive and also the wish to stay longer at home. This creates a health risk, especially when the aged persons live alone. In addition to health problems, a person may fall and be rendered unconscious or injured and may not be able to alert someone. Fall incidents are the most important cause of accidents for elderly people and often result in serious physical and psychological consequences [2].

Health monitoring is currently being achieved through active involvement, e.g., pressing a button worn as a necklace. In many situations, this imposes an important risk factor. Persons may forget to put on the necklace, or may not be able to press the button in emergency situations. The ideal solution is contactless health monitoring, avoiding the need for actions by the person.

Current approaches for remote healthcare monitoring are based on CW Doppler radar [3] or UWB IR radar [4]. They are used just for vital signs detection, especially heartbeat and respiration rate. To the authors' knowledge, the use of radars has not been explored yet for in-door fall detection. Current systems under investigation for contactless fall detection are based on video cameras. This not only causes privacy concerns, but also inaccuracy is an issue, as the number of false positives is still high [5].
In this paper we show the feasibility of using SFCW radar working in the lower microwave frequency range to detect fall incidents while also monitoring the vital signs.

In Section II the radar architecture that was employed is introduced. The experimental results will be discussed in Section III.

\section{RADAR ARCHITECTURE}

In this section we will show that the SFCW radar is the most suitable architecture for fall detection, in combination with vital signs detection. The block diagram of the Quadrature SFCW radar is shown in Fig. 1. The radar is constructed using two wideband antennas, a power splitter, a wideband LNA and an IQ demodulator. In order to demonstrate the functionality, the radar is realised as a board design using off-the-shelf circuits. A version using only a single antenna is currently under development.

An SFCW radar transmits a group of $N$ coherent pulses whose frequencies are increased from pulse to pulse by a fixed frequency increment $\Delta f$ as indicated Fig. 1. The frequency of the $n$-th pulse can be written as:

$$
f_{n}=f_{0}+n \Delta f
$$

where $f_{0}$ is the starting carrier frequency and $\Delta f$ is the frequency step size, that is, the change in frequency from pulse to pulse. Each pulse is $\tau$ seconds wide, and the time interval $T$ between the pulses is adjusted for ambiguous or unambiguous range. Each frequency is constant within each pulse. A group of $N$ pulses, also called burst, is transmitted and received before any processing is initiated to realize a high-resolution measurement of the signal being measured. Since the frequency is constant within the individual pulse, its bandwidth is approximately equal to the inverse of the pulse width. These pulses have narrow bandwidths, thus making the instantaneous bandwidth of the radar narrow.

On the other hand, the overall SFCW radar operation presents an ultra-wideband nature, as opposed to the Doppler $\mathrm{CW}$ radar, allowing distance measurements. Due to its narrowband nature, a pure $\mathrm{CW}$ radar is not able to determine absolute distances. 
Unlike other ultra-wideband architectures, the narrow instantaneous bandwidth combined with the large effective bandwidth (sequentially over many pulses) of the SFCW radar implies that the hardware requirements become less stringent. Lower-speed ADCs and lower level processors can be used. Moreover, the receiver bandwidth is smaller, resulting in lower noise bandwidth and higher signal-to-noise ratio. This increases also the radar sensitivity. Other advantages of SFCW radar are:

- ability to resolve closely spaced targets in range;

- improvement in ranging accuracy;

- reduction of the amount of clutter within the resolution cell;

- reduction of multipath;

- high-resolution ranging profiles;

- aid in target classification;

- increase of the signal-to-clutter ratio.

Moreover, to detect speed, an SFCW radar can exploit the Doppler effect like in a conventional $\mathrm{CW}$ radar. This means that for a good accuracy in speed we do not require a high accuracy in target position like in the case of an impulse radar, where the speed information is obtained dividing space displacement by its time interval. The higher the distance resolution, the wider the bandwidth will be. This means that an SFCW radar can operate with a smaller bandwidth. In fact, for fall detection, a resolution in position of $15 \mathrm{~cm}$ can be considered a proper value to establish if a person has fallen or not. With an SFCW radar, a range resolution of $15 \mathrm{~cm}$ correspond to a total bandwidth of $1 \mathrm{GHz}$ while simultaneously we can detect speed by exploiting the Doppler effect. This contrasts with an impulse radar where for a proper value of speed the range resolution should clearly be smaller than $15 \mathrm{~cm}$ and therefore it would require a wider bandwidth increasing both the complexity and power consumption of the radar transceiver.

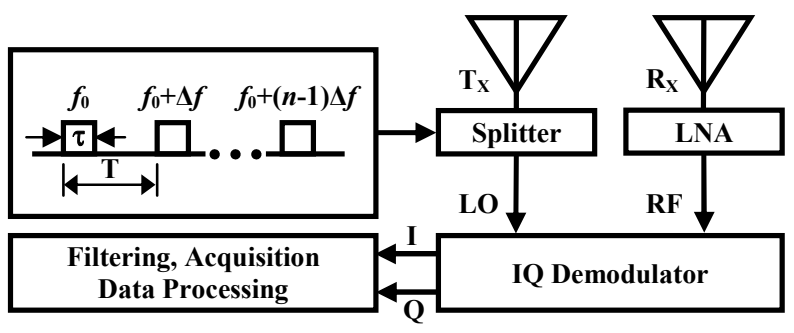

Fig. 1. SFCW Radar System.

\section{EXPERIMENTAL RESULTS}

The radar setup that is used in the experiments is based on above SFCW architecture. $N=121$ pulses are transmitted starting from $1.5 \mathrm{GHz}$ to $2.7 \mathrm{GHz}$ with $\Delta f=10 \mathrm{MHz}$. A transmitted power of $-10 \mathrm{dBm}$ is sent to the antenna. The echo reflected off the target is mixed with the transmitted signal to produce the $I$ and $Q$ components. In particular, the echo reflected from the $n$-th pulse should go back to the receiver and then be mixed with the $n$-th transmitted pulse before a new $f_{n+1}$ pulse is transmitted. Since both transmitted and received $n$-th pulse are close in frequency, the mixing will produce $I$ and $Q$ DC levels (Fig. 2). Since the downconverted signals are at DC, the typical sampling rate is one complex sample per pulse width, making the sample rate lower compared to other ultra wideband radars. It should be noted that a sample rate of ten points per pulse time $\tau$ has been chosen in Fig. 2, just for clarity of the picture. The $I$ and $Q$ samples are related as:

$$
C_{n}=I_{n}+j Q_{n}
$$

where $n$ is the index of the $n$-th frequency in the burst. Applying (2) improves the signal-to-noise ratio by $3 \mathrm{~dB}$.

In order to determine absolute distance, the entire bust should be received and processed. Moreover, the burst interval $\left(N^{*} T\right)$ should be such that the target may be assumed static during a burst. For $N=121$, typical values for $\tau$ and $T$ are respectively $50 \mu$ s and $65 \mu \mathrm{s}$. This involves a burst interval $N^{*} T$ shorter then $8 \mathrm{~ms}$ which is sufficiently small to fulfil the above condition.

The unambiguous and the resolution range, respectively $R$ and $\Delta R$, are determined by the following formulas:

$$
\begin{gathered}
R=\frac{c}{2 \Delta f} \\
\Delta R=\frac{c}{2 N \Delta f} .
\end{gathered}
$$

In our case, $R$ and $\Delta R$, are respectively $15 \mathrm{~m}$ and $12.4 \mathrm{~cm}$.

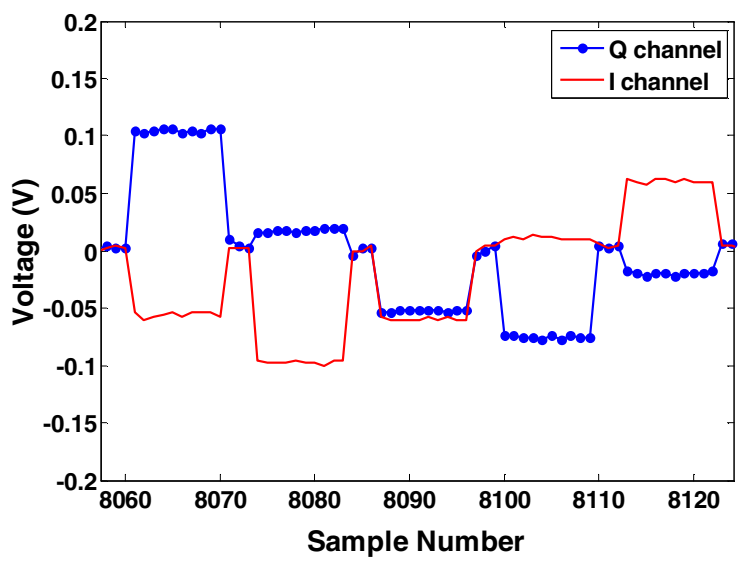

Fig. 2. I and Q DC levels after mixing. 
The first step in data processing of a step-frequency burst is to organize the $C_{n}$ samples of $M$ bursts in a matrix as shown in Table I. Each row represents the $N(=121)$ complex samples of a burst. By IFFT compression to each row of the matrix, the range profile is obtained. It determines the absolute distance of the target each $N^{*} T$ seconds. This is true if the assumption of a static target during burst interval $\left(N^{*} T\right)$ is valid.

In order to detect the position of a person, different measurements have been performed by fixing the radar both on the ceiling and on the wall. Figs. 3 and 4 show the range profile of a standing person when the radar is fixed respectively on the wall and on the ceiling. In both radar positions, the peak corresponding to the standing person is very clear. These results clearly show how the SFCW radar can determine the absolute distance of a target. Moreover it is possible to see that the radar is able to separate the target peak from the static reflections, corresponding to clutter.

Regarding the speed of the target, just one frequency among the $N$ pulses is considered and the Doppler principle is exploited. This means that just one column of the matrix should be considered. In our case the column of the $1.7 \mathrm{GHz}$ frequency has been chosen. This operation is equivalent to having a pure CW Doppler radar working at 1.7 GHz. The relative samples give birth to a signal whose frequency is altered in proportion to the velocity of the target according to the Doppler effect:

$$
f_{d}(t)=2 \frac{f \cdot v(t)}{c}
$$

where $f$ is the frequency of the incident signal $(1.7 \mathrm{GHz}$ in our case), $v(t)$ is the velocity of the moving object, $c$ is the speed of light, and $f_{d}(t)$ is the resulting shift in frequency.

Also in this case, measurements were performed with the radar fixed both on the wall and on the ceiling. The results show clearly the difference between a fall and a normal movement (walking or sitting). During a fall, the speed continuously increases until the sudden moment when the fall is finished. During walking or sitting down, the Doppler signal experiences a controlled movement. More precisely, while a person is sitting down the speed first gradually increases, and then decreases and stops smoothly. During a walk, instead, the speed is quite constant over time. Fig. 5 and Fig. 6 show the speed signal during a fall and a walk respectively. In both situations, the subject was initially in static and standing condition. In the first case, the fall lasts around 0.9 seconds. In this time interval it is clearly seen how the signal frequency (and thus the speed) increases with time and then abruptly stops when the ground is reached. In the second case, the frequency of the signal is quite constant over time.
By applying the IFFT compression first to the rows and then to the columns of the matrix in Table I, the range information and vital signs frequencies of a person in fixed position can be obtained. Fig. 7 shows the respiration rate of a person.

TABLE I

COMPLEX SAMPLES OF DIFFERENT BURSTS IN A MATRIX

\begin{tabular}{|c|c|c|c|c|c|}
\hline burst & $\mathbf{f}_{\mathbf{0}}$ & $\mathbf{f}_{\mathbf{0}}+\mathbf{\Delta} \mathbf{f}$ & $\mathbf{f}_{\mathbf{0}}+\mathbf{2} \boldsymbol{\Delta} \mathbf{f}$ & $\ldots$ & $\mathbf{f}_{\mathbf{0}}+(\mathbf{N}-\mathbf{1}) \mathbf{\Delta f}$ \\
\hline $\mathbf{1}$ & $\mathrm{C}_{11}$ & $\mathrm{C}_{12}$ & $\mathrm{C}_{13}$ & $\ldots$ & $\mathrm{C}_{1 \mathrm{~N}}$ \\
\hline $\mathbf{2}$ & $\mathrm{C}_{21}$ & $\mathrm{C}_{22}$ & $\mathrm{C}_{23}$ & $\ldots$ & $\mathrm{C}_{2 \mathrm{~N}}$ \\
\hline $\mathbf{3}$ & $\mathrm{C}_{31}$ & $\mathrm{C}_{32}$ & $\mathrm{C}_{33}$ & $\ldots$ & $\mathrm{C}_{3 \mathrm{~N}}$ \\
\hline $\mathbf{4}$ & $\mathrm{C}_{41}$ & $\mathrm{C}_{42}$ & $\mathrm{C}_{43}$ & $\ldots$ & $\mathrm{C}_{4 \mathrm{~N}}$ \\
\hline $\boldsymbol{\ldots}$ & $\ldots$ & $\ldots$ & $\ldots$ & $\ldots$ & $\ldots$ \\
\hline $\mathbf{M}$ & $\mathrm{C}_{\mathrm{M} 1}$ & $\mathrm{C}_{\mathrm{M} 2}$ & $\mathrm{C}_{\mathrm{M} 3}$ & $\ldots$ & $\mathrm{C}_{\mathrm{MN}}$ \\
\hline
\end{tabular}

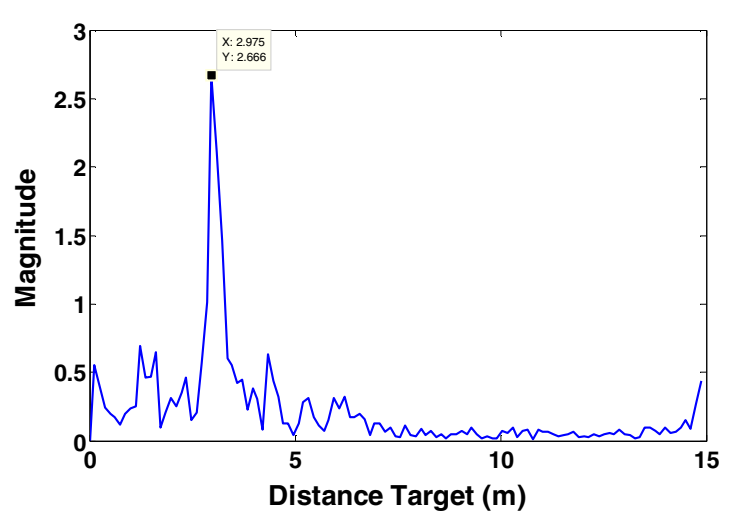

Fig. 3. Range profile of a standing person with the radar fixed on the wall.

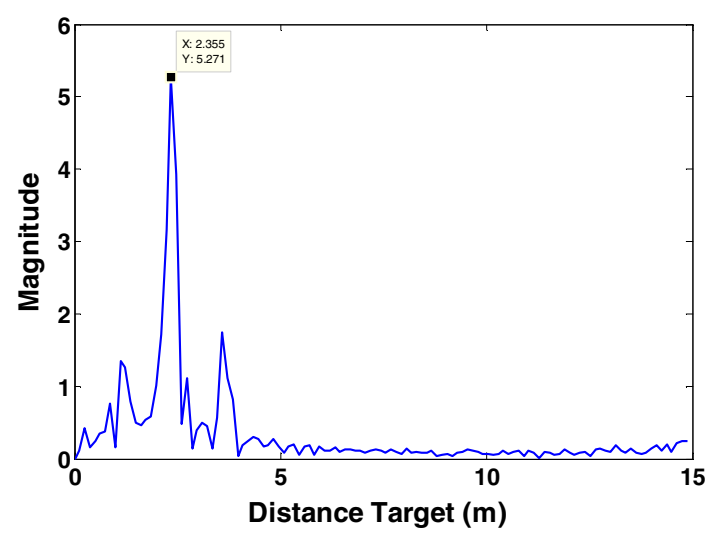

Fig. 4. Range profile of a standing person with the radar fixed on the ceiling. 


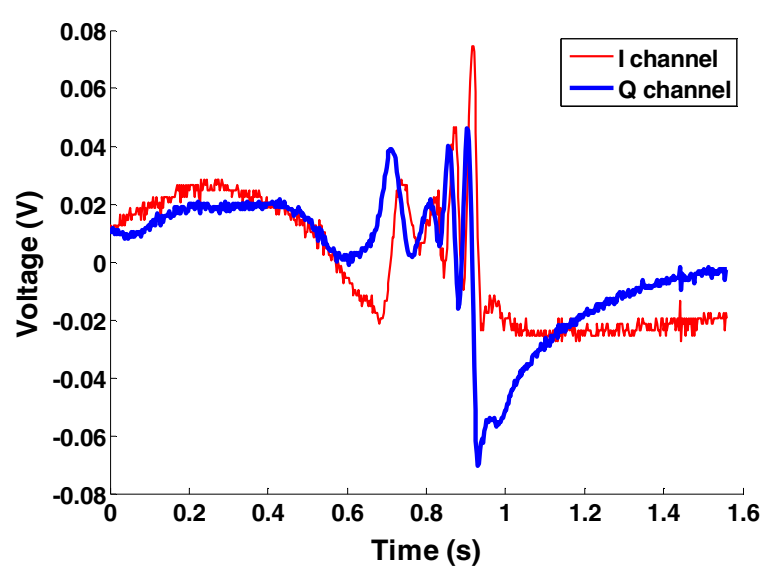

Fig. 5. Speed signal during a fall. The frequency of the signal is proportional to the velocity of the person during the fall.

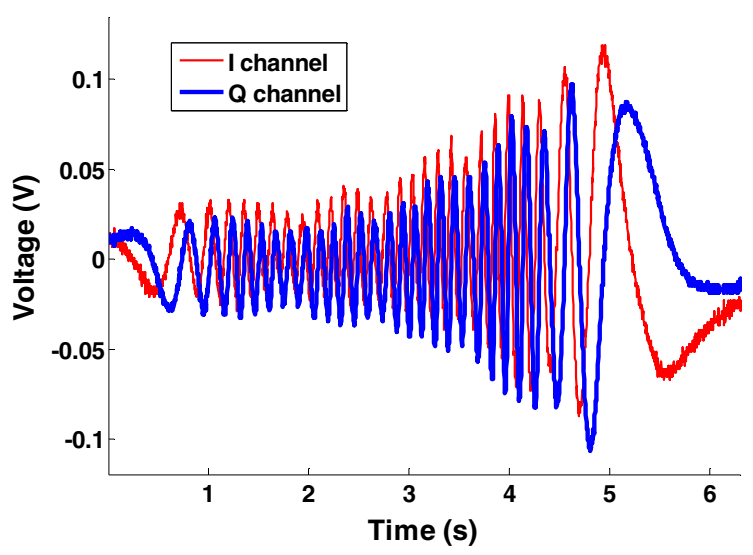

Fig. 6. Speed signal during a walk. The frequency of the signal is proportional to the velocity of the person during the walk.

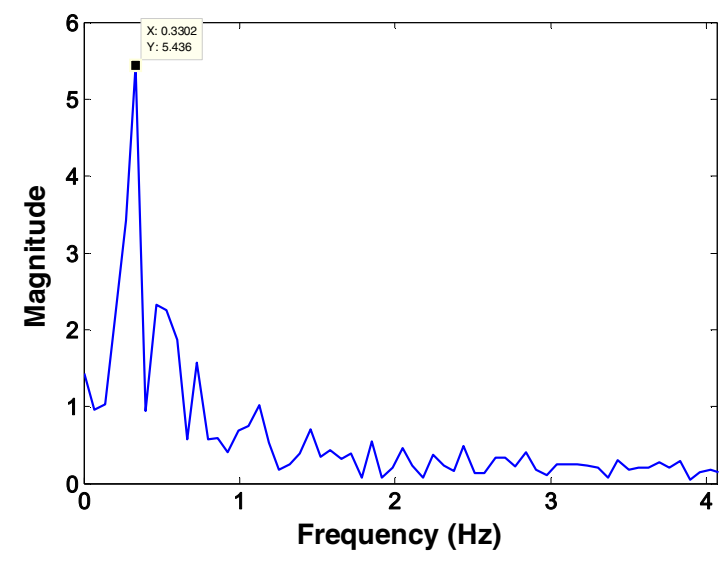

Fig. 7. Respiration rate of a person.

\section{CONCLUSION}

An SFCW radar system has been developed which is capable of fall detection in an indoor environment. The system is inline with the growing need for home health care applications and supervision technology for elderly people living at home. A pure $\mathrm{CW}$ radar can measure the target's velocity without ambiguity, but is not able to determine absolute distances. The presented SFCW radar utilizes different frequencies as timing marks to realize a high resolution system providing the range resolution of wideband systems with the implementation advantages of narrowband systems. This involves a cost effective and an attractive technique to detect fall incidents and to monitor vital signs.

In this paper, for the first time we give a proof of remote indoor fall detection by using a radar. By determining and combining the information about absolute distances and speed of a target, it is possible to distinguish a fall from a normal movement (i.e., walking or sitting down). This concept has been demonstrated by measurements. The same radar architecture is able to monitor vital signs simultaneously.

\section{ACKNOWLEDGEMENTS}

This work has been supported by FWO-Flanders and K.U.Leuven GOA project.

\section{REFERENCES}

[1] O. Boric-Lubecke and V.M. Lubecke, "Wireless house calls: using communications technology for health care and monitoring," IEEE Microwave Magazine, vol. 3, pp. 43-48, Sep. 2002.

[2] P. Haentjens, J. Magaziner, et al., "Excess mortality after hip fracture among older women and men," Annals of Internal Medicine, vol. 152, pp. 380-390, 2010.

[3] A.D. Droitcour, O. Boric-Lubecke, V.M. Lubecke, J. Lin, and G.T.A. Kovac, "Range correlation and I/Q performance benefits in single-chip silicon Doppler radars for noncontact cardiopulmonary monitoring," IEEE Transactions on Microwave Theory and Techniques, vol. 52, no. 3, pp. 838848, March 2004.

[4] I. Y. Immoreev, S. Samkov, and T.-H. Tao, "Short-distance ultra wideband radars," IEEE Aerospace and Electronic Systems Magazine, vol. 20, no. 6, pp. 9-14, June 2005.

[5] G. Debard, J. Van den Bergh, B. Bonroy, M. Deschodt, E. Dejaeger, K. Milisen, T. Goedemé, and B. Vanrumste, "Fallcam: Practical Considerations in Implementing a Camera-based Fall Detection System," International Conference on Positioning and Context-Awareness, 28 May 2009. 